\title{
Comparison of plaque characteristics of small and large subcortical infarctions in the middle cerebral artery territory using high-resolution magnetic resonance vessel wall imaging
}

\author{
Tingting Zhu ${ }^{1,2}$, Lijie Ren ${ }^{3}$, Lei Zhang ${ }^{2,4}$, Yinghui Shao ${ }^{3}$, Liwen Wan ${ }^{2,4}$, Ye $\mathrm{Li}^{2,4}$, Dong Liang ${ }^{2,4}$, \\ Hairong Zheng ${ }^{2,4}$, Xin Liu ${ }^{2,4}$, Na Zhang ${ }^{2,4}$ \\ ${ }^{1}$ Department of Radiology, Tongji Hospital, Tongji Medical College, Huazhong University of Science and Technology, Wuhan, China; ${ }^{2}$ Paul C. \\ Lauterbur Research Center for Biomedical Imaging, Shenzhen Institutes of Advanced Technology, Chinese Academy of Sciences, Shenzhen, China; \\ ${ }^{3}$ Department of Neurology, Shenzhen Second People's Hospital, Shenzhen, China; ${ }^{4}$ CAS Key Laboratory of Health Informatics, Shenzhen Institutes \\ of Advanced Technology, Chinese Academy of Sciences, Shenzhen, China
}

Correspondence to: Na Zhang, PhD. Paul C. Lauterbur Research Center for Biomedical Imaging, Shenzhen Institutes of Advanced Technology, Chinese Academy of Sciences, 1068 Xueyuan Ave., Shenzhen 518055, China. Email: na.zhang@siat.ac.cn.

Background: The characteristics of plaque that ultimately lead to different subcortical infarctions remain unclear. We explored the differences in plaque characteristics between patients with small subcortical infarction (SSI) and large subcortical infarction (LSI) of the middle cerebral artery (MCA) using highresolution magnetic resonance vessel wall imaging (HR-MRVWT).

Methods: The study group comprised 71 patients (mean age, $47.49 \pm 11.5$ years; 55 male) with MCA territory ischemic stroke. Whole-brain HR-MRVWI was performed using a three-dimensional T1-weighted variable-flip-angle turbo spin echo (SPACE) sequence. Patients were divided into SSI and LSI groups based on routine MRI images. Plaque distribution was classified as the superior, inferior, ventral, or dorsal wall of the MCA. The number of quadrants with plaque formation, location of plaque, plaque burden (PB), arterial remodeling pattern (positive or negative), and degree of stenosis were analyzed and compared between groups.

Results: Of the 71 patients, 43 (60.6\%) and 28 (39.4\%) were identified as the SSI and LSI groups, respectively. The proportion of plaques involving only one quadrant was significantly higher in the SSI group, and these plaques were located in the superior or dorsal MCA vessel wall. There was no significant difference between groups in the proportion of plaques involving two or more quadrants, plaque distribution, or PB. Most plaques in both groups showed positive remodeling, and the percentage of remodeling pattern was similar. A significantly higher incidence of low-grade stenosis $(<50 \%)$ was observed in the SSI group.

Conclusions: Both SSI and LSI may be associated with major intracranial artery atherosclerosis, but patients with SSI showed relatively fewer quadrants with plaque formation and a lesser degree of stenosis.

Keywords: Atherosclerotic plaque; ischemic stroke; magnetic resonance vessel wall imaging (MRVWI); subcortical infarction

Submitted Feb 20, 2020. Accepted for publication Jul 21, 2020.

doi: 10.21037/qims-20-310

View this article at: http://dx.doi.org/10.21037/qims-20-310 


\section{Introduction}

Small subcortical infarction (SSI), which often includes 'lacunar infarction,' accounts for approximately $25 \%$ of ischemic cerebral infarctions and usually occurs in the territory of the middle cerebral artery (MCA) $(1,2)$. It is generally believed that SSI is caused by intrinsic diseases of the penetrating arteries themselves $(3,4)$. However, several recent studies have shown that the incidence of large artery atherosclerosis, which is important pathogenesis of large subcortical infarction (LSI), is also high in patients with SSI (5-9). This suggests that in patients with large artery atherosclerosis, the underlying mechanism of SSI may be the same as that causing LSI and could be inferred from the characteristics of the atherosclerotic plaque.

An increasing number of studies have shown that arterial wall morphology and plaque characteristics help distinguish the different mechanisms underlying stroke (10-13). The remodeling pattern (positive or negative), distribution (superior, inferior, ventral or dorsal wall), plaque burden (PB), and vulnerability of intracranial plaque may be associated with the pathogenesis and disease process of different types of infarction (6,14-16). However, the plaque characteristics that ultimately lead to SSI or LSI remain unclear.

High-resolution magnetic resonance vessel wall imaging (HR-MRVWI) has demonstrated its potential to depict large arterial vessel wall lesions and characterize arterial plaques and has proven to be reproducible (17-19). However, few studies have focused on unraveling the differences in plaque characteristics among the stroke subtypes (such as SSI and LSI), which may help determine the cause of subcortical infarction. In the present study, we explored the differences in plaque characteristics in the MCA between patients with LSI and SSI using HR-MRVWI.

\section{Methods}

The data that support the findings of this study are available from the corresponding author upon reasonable request.

\section{Subjects}

From October 2017 to February 2019 at one imaging center, 71 consecutive patients (mean age, $47.49 \pm 11.5$ years; 55 men) with a recent ischemic event (such as one-sided numbness and weakness, etc.) were prospectively enrolled in the study and underwent HR-MRVWI examination within 4 weeks of symptom onset. The general clinical information, including age, sex, and vascular risk factors, such as hypertension, diabetes mellitus, hyperlipidemia, smoking history, and history of transient ischemic attack or stroke, were recorded for each patient. The institutional review board approved this study of Shenzhen Institutes of Advanced Technology, Chinese Academy of Sciences (No. SIAT-IRB-180215-H0204) and written informed consent was obtained from the patient.

All the patients were enrolled according to the following inclusion criteria: (I) first-time acute ischemic stroke located subcortically to and within the unilateral MCA territory as identified by $\mathrm{T} 2$-weighted fluid-attenuated inversion recovery (T2W-FLAIR); (II) ipsilateral internal carotid artery not showing significant stenosis ( $\geq 50 \%$ stenosis), as confirmed by MR angiography, CT angiography or digital subtraction angiography; and (III) no evidence of other causes of stroke (coagulopathy, potential cardioembolic source of disease, Moya-Moya disease, dissection, etc.). Cortical strokes were excluded from this study because cortical infarct often involves more than one cerebral artery territory.

\section{MRI protocols}

MRI examinations were performed using a 3T MR system (MAGNETOM TIM Trio; Siemens Medical Solutions, Erlangen, Germany) with a 32-channel phase array head coil. Routine brain MRI (T1W- and T2W-FLAIR) was initially performed for clinical evaluation of the stroke patients. Whole-brain HR-MRVWI was subsequently performed with a three-dimensional (3D) T1-weighted variable-flip-angle turbo spin echo (SPACE) sequence with the following imaging parameters: repetition time/echo time $=900 / 15 \mathrm{~ms}$, field of view $=170 \times 170 \times 128 \mathrm{~mm}^{3}$, number of slices $=256$, spatial resolution $=0.53 \times 0.53 \times 0.53 \mathrm{~mm}^{3}$ without interpolation, sagittal orientation, generalized autocalibrating partially parallel acquisitions (GRAPPA) accelerated factor $=2$, turbo factor $=52$, bandwidth $=446 \mathrm{~Hz} /$ pixel, and scan time $8 \min 10 \mathrm{~s}$.

\section{Routine MRI assessment}

The diagnosis of MCA territorial infarction was based on previous templates $(20,21)$. The patients were divided into two groups according to the size of the lesion on the T2FLAIR images. (I) The SSI group comprised patients with a maximal axial lesion diameter of $\leq 20 \mathrm{~mm}$ (22). (II) The 
Table 1 General clinical, demographic data of patients with SSI and LSI

\begin{tabular}{lccc}
\hline Variables & SSI $(\mathrm{n}=43)$ & LSI $(\mathrm{n}=28)$ & $\mathrm{P}$ value \\
\hline Age (years) & $47.70 \pm 11.35^{*}$ & $47.14 \pm 11.92^{*}$ & 0.844 \\
Male & $34(79.1 \%)$ & $21(75 \%)$ & 0.689 \\
Hypertension & $26(60.5 \%)$ & $15(53.6 \%)$ & 0.566 \\
Diabetes mellitus & $13(30.2 \%)$ & $6(21.4 \%)$ & 0.408 \\
Hyperlipidemia & $17(39.5 \%)$ & $14(50.0 \%)$ & 0.385 \\
Smoking & $15(34.9 \%)$ & $12(42.9 \%)$ & 0.500 \\
History of stroke & $2(4.7 \%)$ & $2(7.1 \%)$ & 0.660 \\
\hline
\end{tabular}

${ }^{*}$, mean \pm standard deviation. LSI, large subcortical infarction; SSI, small subcortical infarction.

LSI group comprised patients with larger lesions who were not categorized as having SSIs and included large, deep perforator infarcts, border zone infarcts, and superficial perforator infarcts (21).

\section{HR-MRVWI analysis}

All the MRI images were reviewed and reconstructed using the multiplanar reconstruction tool on a workstation (Syngo Multi-Modality Workplace; Siemens Healthcare, Erlangen, Germany) by two neuroradiologists with more than 6 years' experience. A cross-sectional slice of the most stenotic part of the MCA on the ipsilateral side of the cerebral infarction was reconstructed to assess plaque distribution and $\mathrm{PB}$. When there were multiple lesions, the site of maximal stenosis was analyzed. A cross-sectional slice of the ipsilateral normal vessel was reconstructed as a reference slice for calculating the remodeling pattern and degree of stenosis.

The plaque distribution on the cross-sectional image was classified as the superior, inferior, dorsal, or ventral side of the vessel well, according to a previous report (23). In the case of two or more quadrants being involved, the location and number of quadrants with plaque formation were recorded. Using commercial software (VesselMass, Leiden University Medical Center, Leiden, The Netherlands), the contours of the luminal and outer vessel wall boundaries were traced manually on a reconstructed cross-sectional plaque slice and reference slice, respectively. Lumen area (LA) and vessel area (VA) were automatically generated. The PB was calculated as (VA - LA)/VA $\times 100 \%$. The remodeling index (RI) was calculated as the ratio of the VA of the plaque slice to that of the reference slice. Three remodeling patterns were defined according to the RI value: if $\mathrm{RI}>1.05$, it was defined as positive; if $0.95 \leq \mathrm{RI} \leq 1.05$, it was classified as intermediate; and negative if $\mathrm{RI}<0.95$ (16). The degree of stenosis was defined as $\left(1-\mathrm{LA}_{\text {lesion }} / \mathrm{LA}_{\text {reference }}\right)$ $\times 100 \%$, where $\mathrm{LA}_{\text {lesion }}$ and $\mathrm{LA}_{\text {reference }}$ are the LAs derived from a cross-sectional plaque slice and reference slice, respectively. The degree of stenosis was graded as low-grade if stenosis was $<50 \%$, and moderate-to-severe if stenosis was $\geq 50 \%$.

\section{Statistical analysis}

All the statistical analyses were performed using SPSS software (version 25.0, IBM SPSS, Chicago, IL, USA). Fisher's test was performed to evaluate the difference in the percentage of quadrants with plaque formation, $\mathrm{PB}$, remodeling pattern, and degree of stenosis between the SSI and LSI groups. The Wilcoxon signed-rank test was performed to determine the difference in $\mathrm{PB}$ between the two groups. A two-tailed $\mathrm{P}$ value $\leq 0.05$ was considered to indicate a significant difference.

\section{Results}

\section{General clinical characteristics}

Of the 71 patients, $43(60.6 \%)$ were assigned to the SSI group, and 28 (39.4\%) to the LSI group. The general clinical demographics of the patients are presented in Table 1 . No significant difference in risk factors was found between the two groups.

\section{Plaque distribution in the MCA}

For the SSI group, no eccentric or concentric wall 
Table 2 Comparison of the number of quadrants with plaque formation in the SSI and LSI groups

\begin{tabular}{lcccc}
\hline Group & 1 quadrant & 2 quadrants & 3 quadrants & 4 quadrants \\
\hline SSI $(n=38)^{*}$ & $13(34.2 \%)$ & $17(44.7 \%)$ & $3(7.9 \%)$ & $5(13.2 \%)$ \\
LSI $(n=28)^{*}$ & $2(7.1 \%)$ & $19(67.9 \%)$ & $4(14.3 \%)$ & $3(10.7 \%)$ \\
P value & 0.006 & 0.060 & 0.408 & 0.763 \\
\hline
\end{tabular}

${ }^{*}$, number of plaques. LSI, large subcortical infarction; SSI, small subcortical infarction.

Table 3 Comparison of plaque distribution in the SSI and LSI groups

\begin{tabular}{lcccc}
\hline \multirow{2}{*}{ Group } & \multicolumn{3}{c}{ Vessel wall location } \\
\cline { 2 - 5 } & Superior & Inferior & Ventral & Dorsal \\
\hline $\mathrm{SSI}(\mathrm{n}=76)^{*}$ & $21(27.6 \%)$ & $19(25.0 \%)$ & $22(28.9 \%)$ & $14(18.4 \%)$ \\
$\mathrm{LSI}(\mathrm{n}=64)^{*}$ & $18(28.1 \%)$ & $14(21.9 \%)$ & $21(32.8 \%)$ & $11(17.2 \%)$ \\
$\mathrm{P}$ values & 0.948 & 0.664 & 0.622 & 0.849 \\
\hline
\end{tabular}

*, number of quadrants. LSI, large subcortical infarction; SSI, small subcortical infarction.

thickening was found in 5 of the 43 patients (11.6\%). In the LSI group, all patients had eccentric or non-eccentric wall thickening of the MCA on the ipsilateral side of the cerebral infarction. A comparison of the number of quadrants with plaque formation and location of plaque distribution between the SSI group and LSI group is given in Tables 2 and 3, respectively. For the SSI group, the plaque in 13 patients involved only one quadrant, and the proportion was significantly higher than in the LSI group (34.2 vs. 7.1\%, $\mathrm{P}=0.006)$. Figure 1 shows representative HR-MRVWI images of two patients with SSI and LSI, respectively. The plaque in both patients involved only one quadrant.

Furthermore, the 13 plaques were more frequently located in the superior (46.2\%) and dorsal (38.5\%) sides of the vessel wall. The occurrence of plaques involving two or more quadrants was approximately the same in the two groups, and plaques involving two quadrants were the most common distribution pattern in both groups: 17 and 19 plaques in SSI and LSI groups, respectively (Table 2). The plaque distribution in four quadrants was similar between the two groups. The ventral side was a relatively commonly involved quadrant, whereas the dorsal side was a relatively rarely involved quadrant.

\section{$P B$, remodeling pattern, and stenosis degree}

There was no significant difference in $\mathrm{PB}$ between the SSI and LSI groups $(0.75 \pm 0.12$ vs. $0.79 \pm 0.10, \mathrm{P}=0.162)$. The remodeling pattern and degree of stenosis between the two groups are shown in Figure 2. More than half of the plaques exhibited positive remodeling at a rate of $52.6 \%$ and $53.6 \%$ for the SSI and LSI groups, respectively. There was no significant difference in the percentages of positive, negative, and intermediate remodeling patterns between the two groups ( $\mathrm{P}=0.940,0.447$, and 0.336 , respectively). In the LSI group, more patients showed moderate-tosevere vascular stenosis ( $\geq 50 \%$ stenosis), whereas low-grade stenosis ( $<50 \%$ stenosis) was predominant in the SSI group (Figure 3). A significantly higher incidence of low-grade stenosis was found in the SSI group compared with the LSI group $(68.4 \%$ vs. $42.9 \%, \mathrm{P}=0.037)$.

\section{Discussion}

In the present study, approximately $11.6 \%$ of the patients with SSI had no abnormality of the MCA, from which the lenticulostriate arteries are derived. Therefore, the lesions in these cases may be classical lacunar infarctions that are independent of MCA disease, such as would be caused by lipohyalinosis of the perforating arteries. In the remaining $88.4 \%$ of the patients with SSI, eccentric or non-eccentric wall thickening was present in the ipsilateral MCA. This percentage was higher (45.6-72.2\%) than that reported in previous studies $(5,24,25)$. Such a high prevalence may be due to the use of a novel HR-MRVWI and the different subjects included in the study. Advanced 3D HR-MRVWI 


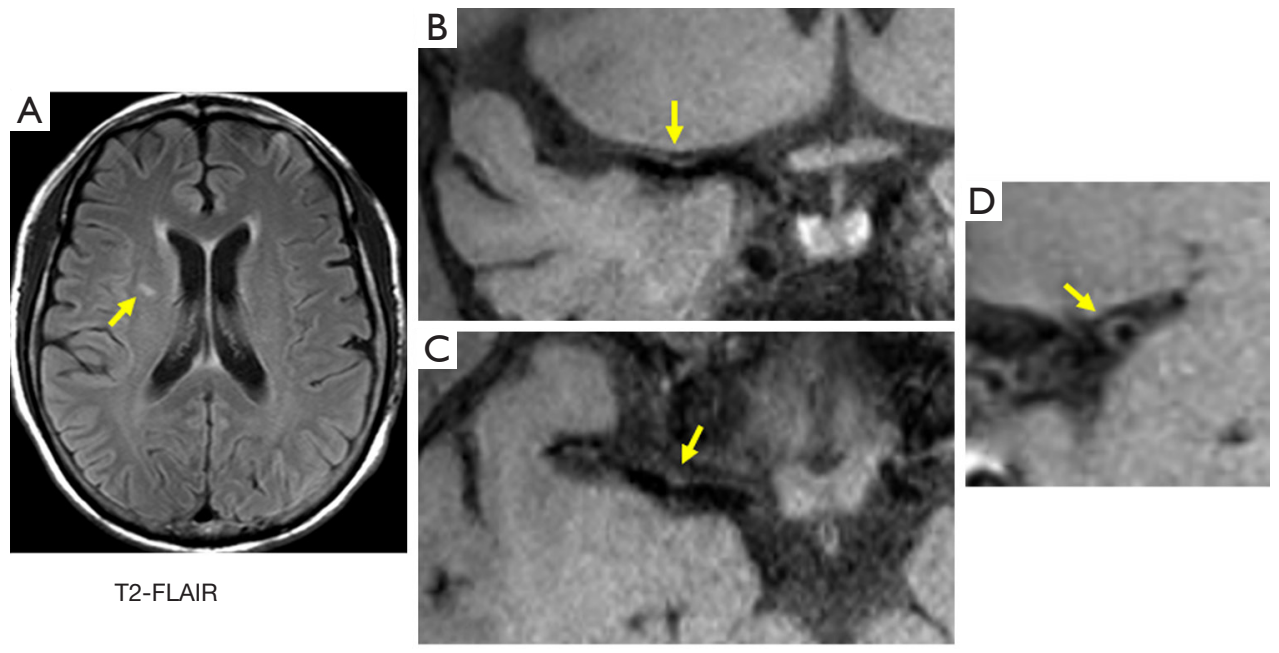

HR-MRVWI

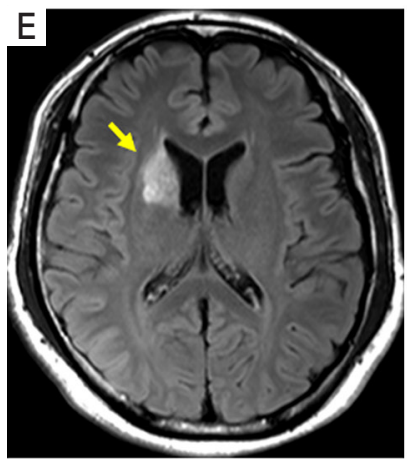

T2-FLAIR
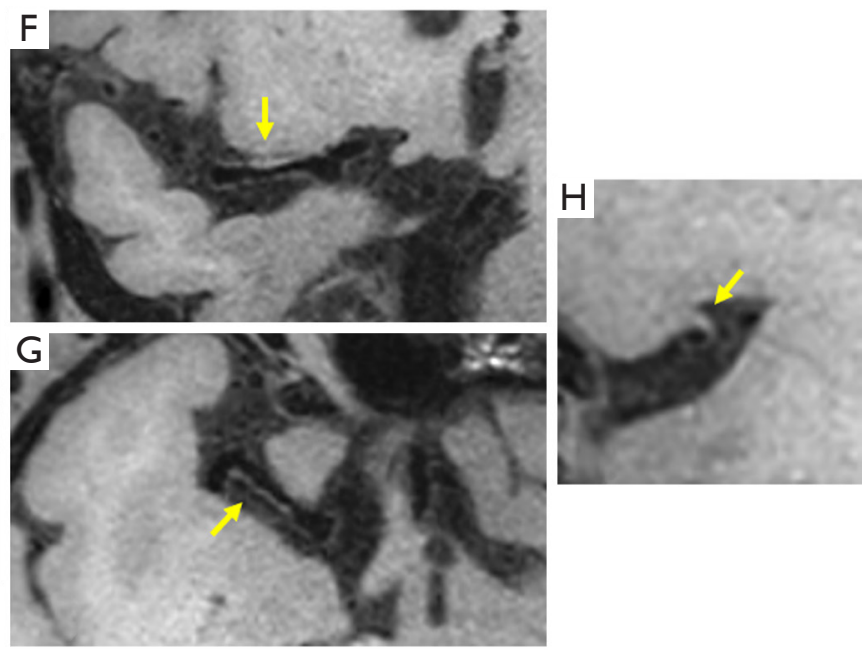

HR-MRVWI

Figure 1 Representative MR images for a patient with SSI (A,B,C,D) and another with LSI (E,F,G,H). T2-FLAIR images (A,E) show a subcortical infarction of the right middle cerebral artery (MCA) territory (arrows). Plaque (arrows) was detected in the ipsilateral MCA on HR-MRVWI images (B,C,F,G) for each patient. The cross-sectional images (D,H) of the plaques (arrows) of both patients show involvement in only one quadrant, specifically ventral for SSI (D) and superior for LSI (H). FLAIR, fluid-attenuated inversion recovery; HR-MRVWI, high-resolution magnetic resonance vessel wall imaging; LSI, large subcortical infarction; SSI, small subcortical infarction.

can identify intracranial plaques that are missed on conventional angiographic imaging. Also, the patients in this study had symptomatic ischemia, and fibrohyalinosis of the arterioles is mainly associated with asymptomatic lacunes $(25,26)$. In a previous study $(6)$, only patients without stenosis of the MCA confirmed by MRA were included. We included patients with symptomatic subcortical ischemia confirmed by MRI, regardless of whether the MRA showed stenosis or not. Our results suggested that atherosclerosis of the MCA may be more prevalent among patients with SSI than previously believed.

SSI caused by a focal atherosclerotic plaque of the MCA trunk may have the following possible mechanisms: (I) the origin of a perforating artery is occluded by the atherosclerotic lesions in the MCA; (II) small embolic particles from the MCA are deposited in a perforating 


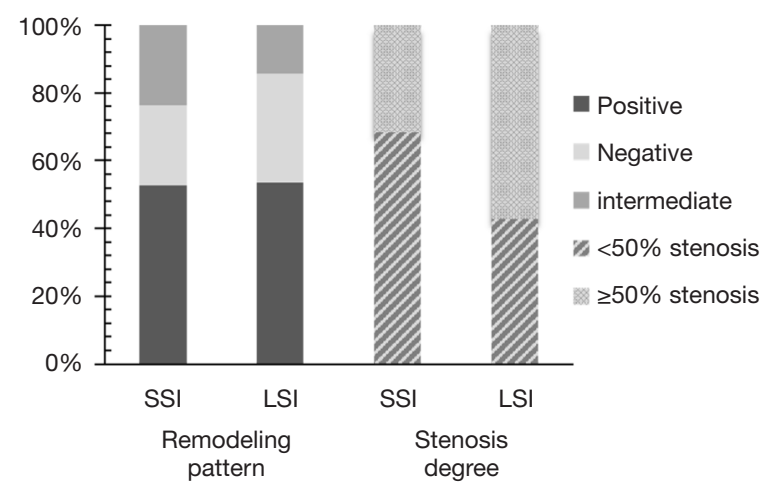

Figure 2 Frequency of positive, intermediate, and negative remodeling patterns and frequency of $<50 \%$ and $\geq 50 \%$ stenosis. LSI, large subcortical infarction; SSI, small subcortical infarction.

artery (27). Microanatomy studies suggest that most of the penetrating branches of the MCA arise from the dorsalsuperior part of the wall (28). Our study revealed that the SSI group had a higher proportion of plaque that involved only one quadrant of the wall. Also, this plaque appeared to be more frequently located in the superior (46.2\%) and dorsal $(38.5 \%)$ walls, thus being more likely to cause an infarct by direct occlusion of the perforating artery. The patients with LSI rarely had small-scale plaques that involved only one quadrant. Our findings suggested that SSI may be associated with MCA atherosclerosis, and plaque distribution may play an important role. Also, atherosclerotic lesions and fibrohyalinosis often coexist (4), but the walls of the perforator artery in patients with SSI could not be directly observed to confirm our hypothesis. The observation of diseased perforators depends on the further development of imaging technology.

Our results did not suggest an association of stroke type concerning involved quadrants or perforator arteries. Also, the ventral side of the vessel wall was a commonly involved quadrant in both groups. The results of other research and our studies show the same trend for the location of plaques (23). The plaque distribution in the MCA in both groups appeared to follow the same pattern as in coronary atherosclerosis, which is opposite the ostia of the penetrating arteries (29). This may be a protective mechanism. We observed that eccentric plaque involving two quadrants was most common in both groups, a finding that was consistent with a previous study showing that eccentric wall thickening is a characteristic of intracranial atherosclerotic plaque (30). However, in the current study, concentric plaques involving four quadrants were also found in the MCA, which is consistent with findings from Dieleman et al.'s study (31).

Previous studies have shown that symptomatic MCA plaques have a greater wall area and prevalence of positive remodeling, compared with asymptomatic plaques $(32,33)$. $\mathrm{PB} \geq 77 \%$ on HR-MRVWI has proven to be a good predictor of the culprit and non-culprit lesions in the MCA (34). We found PB $>70 \%$ in both groups, which may relate to the recruitment of symptomatic patients and the measurement of maximum $\mathrm{PB}$ in each group. These measured plaques on the ipsilateral side to an ischemic stroke are usually identified as culprit plaque in other studies. Similar results have been obtained in the study of the coronary artery, where $\mathrm{PB} \geq 70 \%$ is a predictor of future cardiovascular events (35).

Also, PB was similar between the SSI and LSI groups, suggesting that SSI may not be a mild form of LSI in intracranial atherosclerosis. In the early stage of atherosclerosis, the vascular lumen is usually preserved by outward remodeling. Positive remodeling of the internal carotid artery can preserve the lumen, accounting for $62 \%$ of the VA at the plaque location (36). Although positive remodeling limits the hemodynamic effects of vascular stenosis, it may be associated with increased plaque vulnerability. In our study, positive remodeling of the MCA was observed in more than half of the patients in each group, but particularly in those with SSI; $68.4 \%$ of the patients showed low-grade stenosis, even when the $\mathrm{PB}$ was as high as $75 \%$. Our HR-MRVWI findings were consistent with luminal changes in the internal carotid artery.

We showed that patients with low-grade stenosis accounted for the largest percentage in the SSI group. Previous reports have shown that symptomatic stenosis of the MCA is greater than $50 \%$ (37). Our results extend beyond those of previous studies. Although mild stenosis of a major intracranial artery may not be sufficient to affect the entire vascular territory, the specific location of atherosclerotic plaque may obstruct the blood flow of a perforator artery and lead to smaller infarctions. When the penetrating artery is not blocked, collateral circulation can be established, and newly generated collateral arteries can supply the territory. The territory of the MCA can then endure long-term ischemia, even if the MCA is completely occluded (38). This may be the reason why some patients with SSI showed moderate-to-severe stenosis of the MCA. However, more patients showed moderate-to-severe vascular stenosis in the LSI group. This may be due to inadequate collateral circulation and impaired cerebral 

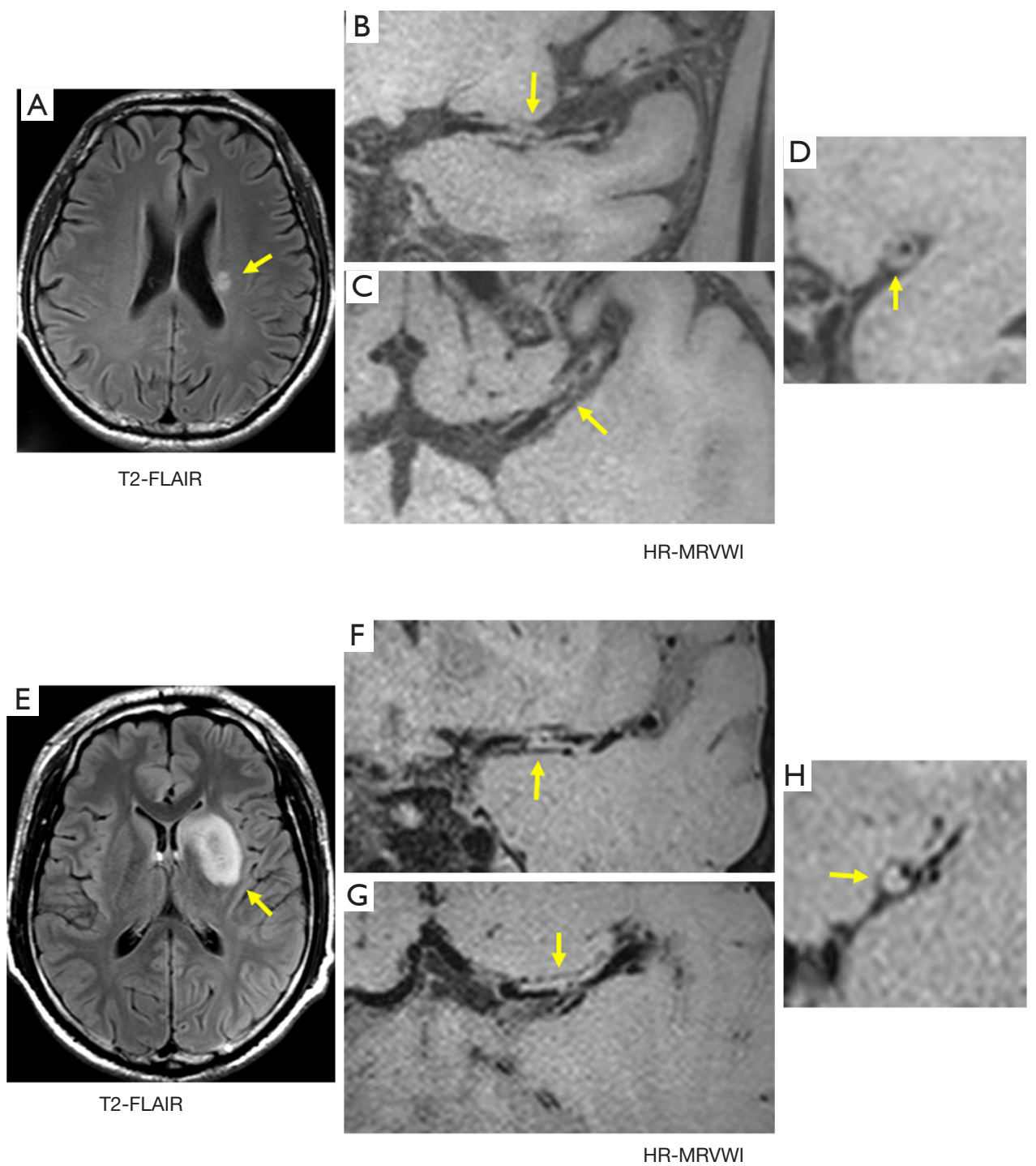

Figure 3 Representative MR images for a patient with SSI (A,B,C,D) and another with LSI (E,F,G,H), respectively. T2-FLAIR images $(\mathrm{A}, \mathrm{E})$ show a subcortical infarction of the left middle cerebral artery (MCA) territory (arrows). Plaque (arrows) was detected in the ipsilateral MCA on HR-MRVWI images (B,C,F,G) for each patient. The cross-sectional images (D,H) of the plaques (arrows) of both patients show involvement of three quadrants, but the plaque in the patient with LSI (H) caused more stenosis than in the patient with SSI (D). FLAIR, fluid-attenuated inversion recovery; HR-MRVWI, high-resolution magnetic resonance vessel wall imaging; LSI, large subcortical infarction; SSI, small subcortical infarction.

perfusion because of hypoperfusion, which leads to border zone infarction, resulting in a larger infarction. Also, the superficial cortex can sometimes remain perfused through collateral circulation, with the patient presenting only with asymptomatic radiological subcortical infarctions. The ostia of multiple penetrating arteries can be blocked by plaque, and larger infarctions of the regions of the perforator artery can also occur, even if the lumen shows low-grade narrowing. In general, hypoperfusion due to severe stenosis may be the primary cause of infarction in patients with moderate-to-severe vascular stenosis, and the specific distribution of plaque leading to blockage of the perforating artery's orifice may be the main cause of infarction in other patients with low-grade stenosis. Therefore, the detection 
of such lesions with HR-MRVWI, even in patients with mild stenosis of the MCA, is of great importance for revealing possible mechanisms of stroke in clinical practice.

This study had several limitations. First, the sample size was relatively small and was in the symptomatic cohort only. Further studies with a larger symptomatic and asymptomatic cohort are needed to determine the complementary value of plaque characteristics to confirm the association of MCA plaque features and any type of stroke in addition to LSI versus SSI. Second, the ostia of the penetrating arteries could not be visualized to prove our hypothesis, as mentioned earlier directly. And there is the possibility of including patients whose infarcts were caused by atherosclerosis of the aortic arch because we did not evaluate aortic arch angiographic images before the study. Third, in the acute stage of stroke, it would be difficult to distinguish atherosclerotic from embolic occlusion when intracranial arterial occlusion is observed. Even if the results of the cardiac assessment are within the normal range, the possibility of cardio-embolism cannot be completely ruled out. Fourth, plaque enhancement and intraplaque hemorrhage, both important features, were not analyzed because we did not have post-contrast HR-MRVWI images. Fifth, we only focused on the most stenotic area for the cross-sectional analysis. Given the roles of eccentricity and remodeling pattern, other areas of the plaque, such as greatest thickness, site of greatest remodeling, greatest eccentricity, etc. could be just as relevant. These also should be considered and evaluated in future studies. Last, because of the lack of perfusion imaging, the findings of a CBF study cannot be presented to confirm our speculation that adequate collateral circulation leads to smaller infarcts even if the patient has severe stenosis. This is warranted in subsequent research.

\section{Conclusions}

In conclusion, both SSI and LSI may be associated with major intracranial arterial atherosclerosis, but patients with SSI show relatively fewer plaque-involved quadrants and a lesser degree of stenosis.

\section{Acknowledgments}

Funding: This work was supported in part by the National Natural Science Foundation of China (81830056 and 81801691), Natural Science Foundation of Guangdong Province (2018A030313204), Guangdong Innovation
Platform of Translational Research for Cerebrovascular Diseases, and Shenzhen Basic Research Program (JCYJ20180302145700745 and JCYJ20170413161350892).

\section{Footnote}

Conflicts of Interest: All authors have completed the ICMJE uniform disclosure form (available at http://dx.doi. org/10.21037/qims-20-310). The authors have no conflicts of interest to declare.

Ethical Statement: The institutional review board approved this study of Shenzhen Institutes of Advanced Technology, Chinese Academy of Sciences (No. SIAT-IRB180215-H0204) and written informed consent was obtained from the patient for publication of this study and any accompanying images.

Open Access Statement: This is an Open Access article distributed in accordance with the Creative Commons Attribution-NonCommercial-NoDerivs 4.0 International License (CC BY-NC-ND 4.0), which permits the noncommercial replication and distribution of the article with the strict proviso that no changes or edits are made and the original work is properly cited (including links to both the formal publication through the relevant DOI and the license). See: https://creativecommons.org/licenses/by-nc-nd/4.0/.

\section{References}

1. Moran C, Phan TG, Srikanth VK. Cerebral small vessel disease: a review of clinical, radiological, and histopathological phenotypes. Int J Stroke 2012;7:36-46.

2. Pantoni L. Cerebral small vessel disease: from pathogenesis and clinical characteristics to therapeutic challenges. Lancet Neurol 2010;9:689-701.

3. Caplan LR. Intracranial branch atheromatous disease: a neglected, understudied, and underused concept. Neurology 1989;39:1246-50.

4. Fisher CM. Lacunes: small, deep cerebral infarcts. Neurology 1965;15:774-84.

5. Xu WH, Li ML, Niu JW, Feng F, Jin ZY, Gao S. Intracranial artery atherosclerosis and lumen dilation in cerebral small-vessel diseases: a high-resolution MRI Study. CNS Neurosci Ther 2014;20:364-7.

6. Zou XD, Chung YC, Zhang L, Han Y, Yang Q, Jia J. Middle Cerebral Artery Atherosclerotic Plaques in Recent Small Subcortical Infarction: A Three-Dimensional High- 
resolution MR Study. Biomed Res Int 2015;2015:540217.

7. Kwan MW, Mak W, Cheung RT, Ho SL. Ischemic stroke related to intracranial branch atheromatous disease and comparison with large and small artery diseases. J Neurol Sci 2011;303:80-4.

8. Nah HW, Kang DW, Kwon SU, Kim JS. Diversity of single small subcortical infarctions according to infarct location and parent artery disease: analysis of indicators for small vessel disease and atherosclerosis. Stroke 2010;41:2822-7.

9. Petrone L, Nannoni S, Del Bene A, Palumbo V, Inzitari D. Branch Atheromatous Disease: A Clinically Meaningful, Yet Unproven Concept. Cerebrovasc Dis 2016;41:87-95.

10. López-Olóriz J, Lopez-Cancio E, Arenillas JF, Hernandez M, Jimenez M, Dorado L, Barrios M, Soriano-Raya JJ, Miralbell J, Caceres C, Fores R, Pera G, Davalos A, Mataro M. Asymptomatic cervicocerebral atherosclerosis, intracranial vascular resistance and cognition: the AsIAneuropsychology study. Atherosclerosis 2013;230:330-5

11. Klein IF, Labreuche J, Lavallee PC, Mazighi M, Duyckaerts C, Hauw JJ, Amarenco P. Is moderate atherosclerotic stenosis in the middle cerebral artery a cause of or a coincidental finding in ischemic stroke? Cerebrovasc Dis 2010;29:140-5.

12. Terasawa Y, Sakai K, Komatsu T, Sakuta K, Omoto S, Mitsumura H, Iguchi Y. Microbleeds of Lacunar Infarction and Middle Cerebral Artery Flow Velocity of Branch Atheromatous Disease Are Essential Factors of Stroke Etiology. Eur Neurol 2019;81:19-23.

13. Shen M, Gao P, Zhang Q, Jing L, Yan H, Li H. Middle Cerebral Artery Atherosclerosis and Deep Subcortical Infarction: A 3T Magnetic Resonance Vessel Wall Imaging Study. J Stroke Cerebrovasc Dis 2018;27:3387-92.

14. van der Kolk AG, Zwanenburg JJ, Brundel M, Biessels GJ, Visser F, Luijten PR, Hendrikse J. Distribution and natural course of intracranial vessel wall lesions in patients with ischemic stroke or TIA at 7.0 Tesla MRI. Eur Radiol 2015;25:1692-700.

15. Degnan AJ, Gallagher G, Teng Z, Lu J, Liu Q, Gillard JH. MR angiography and imaging for the evaluation of middle cerebral artery atherosclerotic disease. AJNR Am J Neuroradiol 2012;33:1427-35.

16. Qiao Y, Anwar Z, Intrapiromkul J, Liu L, Zeiler SR, Leigh R, Zhang Y, Guallar E, Wasserman BA. Patterns and Implications of Intracranial Arterial Remodeling in Stroke Patients. Stroke 2016;47:434-40.

17. Mandell DM, Mossa-Basha M, Qiao Y, Hess CP, Hui F, Matouk C, Johnson MH, Daemen MJ, Vossough A,
Edjlali M, Saloner D, Ansari SA, Wasserman BA, Mikulis DJ. Intracranial Vessel Wall MRI: Principles and Expert Consensus Recommendations of the American Society of Neuroradiology. AJNR Am J Neuroradiol 2017;38:218-29.

18. Fan Z, Yang Q, Deng Z, Li Y, Bi X, Song S, Li D. Wholebrain intracranial vessel wall imaging at 3 Tesla using cerebrospinal fluid-attenuated T1-weighted 3D turbo spin echo. Magn Reson Med 2017;77:1142-50.

19. Zhang N, Zhang F, Deng Z, Yang Q, Diniz MA, Song SS, Schlick KH, Marcel Maya M, Gonzalez N, Li D, Zheng H, Liu X, Fan Z. 3D whole-brain vessel wall cardiovascular magnetic resonance imaging: a study on the reliability in the quantification of intracranial vessel dimensions. J Cardiovasc Magn Reson 2018;20:39.

20. Tatu L, Moulin T, Bogousslavsky J, Duvernoy H. Arterial territories of the human brain: cerebral hemispheres. Neurology 1998;50:1699-708.

21. Bogousslavsky J, Regli F. Centrum ovale infarcts: subcortical infarction in the superficial territory of the middle cerebral artery. Neurology 1992;42:1992-8.

22. Wardlaw JM. What causes lacunar stroke? J Neurol Neurosurg Psychiatry 2005;76:617-9.

23. Xu WH, Li ML, Gao S, Ni J, Zhou LX, Yao M, Peng B, Feng F, Jin ZY, Cui LY. Plaque distribution of stenotic middle cerebral artery and its clinical relevance. Stroke 2011;42:2957-9.

24. Chung JW, Kim BJ, Sohn CH, Yoon BW, Lee SH. Branch atheromatous plaque: a major cause of lacunar infarction (high-resolution MRI study). Cerebrovasc Dis Extra 2012;2:36-44.

25. Fisher CM. The arterial lesions underlying lacunes. Acta Neuropathol 1968;12:1-15.

26. Fisher CM. Capsular infarcts: the underlying vascular lesions. Archives of neurology 1979;36:65-73.

27. Caplan LR. Lacunar infarction and small vessel disease: pathology and pathophysiology. J Stroke 2015;17:2-6.

28. Vincentelli F, Caruso G, Andriamamonjy C, Rabehanta P, Graziani N, Grisoli F, Gouaze A, Vigouroux RP. Micro-anatomy of collateral perforating branches of the middle cerebral artery. Neurochirurgie 1990;36:3-14; discussion 14-5.

29. Watanabe H, Yoshida K, Akasaka T, Hozumi T, Yoshikawa J. Intravascular ultrasound assessment of plaque distribution in the ostium of the left anterior descending coronary artery. Am J Cardiol 1996;78:827-9.

30. Swartz RH, Bhuta SS, Farb RI, Agid R, Willinsky RA, Terbrugge KG, Butany J, Wasserman BA, Johnstone DM, Silver FL, Mikulis DJ. Intracranial arterial wall imaging 
using high-resolution 3-tesla contrast-enhanced MRI.

Neurology 2009;72:627-34.

31. Dieleman N, van der Kolk AG, van Veluw SJ, Frijns CJ, Harteveld AA, Luijten PR, Hendrikse J. Patterns of intracranial vessel wall changes in relation to ischemic infarcts. Neurology 2014;83:1316-20.

32. Chung GH, Kwak HS, Hwang SB, Jin GY. High resolution $\mathrm{MR}$ imaging in patients with symptomatic middle cerebral artery stenosis. Eur J Radiol 2012;81:4069-74.

33. Xu WH, Li ML, Gao S, Ni J, Zhou LX, Yao M, Peng $\mathrm{B}$, Feng F, Jin ZY, Cui LY. In vivo high-resolution MR imaging of symptomatic and asymptomatic middle cerebral artery atherosclerotic stenosis. Atherosclerosis 2010;212:507-11.

34. Teng Z, Peng W, Zhan Q, Zhang X, Liu Q, Chen S, Tian X, Chen L, Brown AJ, Graves MJ, Gillard JH, Lu J. An assessment on the incremental value of high-resolution magnetic resonance imaging to identify culprit plaques in atherosclerotic disease of the middle cerebral artery. Eur Radiol 2016;26:2206-14.

Cite this article as: Zhu T, Ren L, Zhang L, Shao Y, Wan L, Li Y, Liang D, Zheng H, Liu X, Zhang N. Comparison of plaque characteristics of small and large subcortical infarctions in the middle cerebral artery territory using high-resolution magnetic resonance vessel wall imaging. Quant Imaging Med Surg 2021;11(1):57-66. doi: 10.21037/qims-20-310
35. Calvert PA, Obaid DR, O'Sullivan M, Shapiro LM, McNab D, Densem CG, Schofield PM, Braganza D, Clarke SC, Ray KK, West NE, Bennett MR. Association between IVUS findings and adverse outcomes in patients with coronary artery disease: the VIVA (VH-IVUS in Vulnerable Atherosclerosis) Study. JACC Cardiovasc Imaging 2011;4:894-901.

36. Astor BC, Sharrett AR, Coresh J, Chambless LE, Wasserman BA. Remodeling of carotid arteries detected with MR imaging: atherosclerosis risk in communities carotid MRI study. Radiology 2010;256:879-86.

37. Qiao Y, Zeiler SR, Mirbagheri S, Leigh R, Urrutia V, Wityk R, Wasserman BA. Intracranial plaque enhancement in patients with cerebrovascular events on high-spatialresolution MR images. Radiology 2014;271:534-42.

38. Chen H, Hong H, Liu D, Xu G, Wang Y, Zeng J, Zhang R, Liu X. Lesion patterns and mechanism of cerebral infarction caused by severe atherosclerotic intracranial internal carotid artery stenosis. J Neurol Sci 2011;307:79-85. 